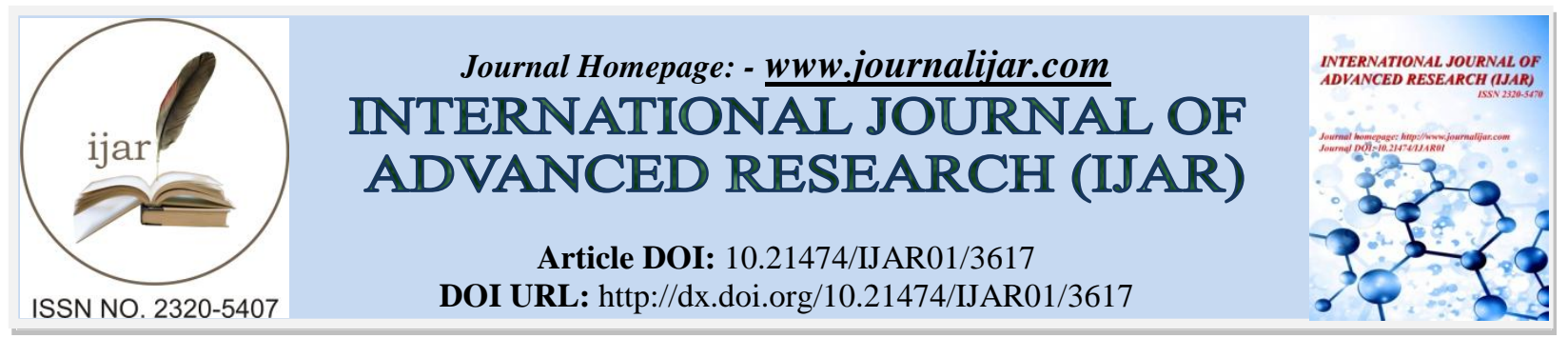

RESEARCH ARTICLE

\title{
INTRAUTERINE INSEMINATION OF SEMINAL PLASMA AT THETIME OF OVUM PICKUP: RELATIONSHIP WITH SUCCESSFUL OUTCOME.
}

Mahmoud H. Ibrahim, MD

Minia Infertility Research Unit, Department of Obstetrics and Gynecology, Minia University Hospital, El-Minia, Egypt.

\section{Manuscript Info}

Manuscript History

Received: 07 January 2017

Final Accepted: 01 February 2017

Published: March 2017

\section{Abstract}

There is a debate about the role of seminal plasma for improved implantation rate and pregnancy rate during in vitro fertilization.

Objective:-The aim of this study is to investigate whether the intrauterine application of SP at the time of the ovum pick-up has any influence on the pregnancy rates in IVF or not.

Methods:- This prospective double-blind placebocontrolled randomized study

Was conducted to compare two groups. The first group represents the cases whom seminal plasma insemination was done and the other group no plasma insemination but placebo. Clinical pregnancy rate and implantation rate were the outcomes.

Results:- 400 patients were randomized after fulfilling the inclusion criteria.200 patients were included in the study group and 200 patients were included in the control group. Our results found no statistically significant difference in the two groups regarding implantation rate or pregnancy rate $(57.3 \%$ versus $49.7 \%, \mathrm{p}$ value $=039)$ and $(39.2 \%$ versus $31.9 \%$, $\mathrm{p}$ value $=0.69$ ).

Conclusion:- Intrauterine insemination of seminal fluid at the time of ovum pickup is unlikely to increase the pregnancy rate.... 


\section{Introduction:-}

Although the gradual increase in the success of reproduction, over the years, many couples also had been left frustrated following repeated failed attempts.Only 20 to $25 \%$ of the transferred embryos lead to a pregnancy.Endometrial function is a limiting factor of the pregnancy[1]. This percentage cannot be further increased by the improvement of the embryo transfer technique and culture conditions or by an optimal selection of embryos, the endometrium is receptive to implantation in the mid-luteal phase during the so-called "implantation window". Its duration is determined by the sex hormones which regulate the expression of several cytokines., endometrial function and receptivity have been accepted to be the major limiting factors of IVF pregnancy rate[2].Human endometrium is a complex, multicellular tissue that is regulated by steroid hormones (estrogens, progesterone, androgens and glucocorticoids) and has different characteristics in the various phases of the menstrual cycle.These changes include restructuring of the cellular architecture, expression of specific cell-surface molecules as well as a secretion of biologically active factors such as cytokines, chemokines and growth factors. The process of the regeneration of the endometrium is currently viewed as a process of cell proliferation and a consequent differentiation of endometrial multipotent stem cells[3]. The presence and the characterization of multipotent stromal stem cells, epithelial progenitor cells and endothelial progenitor cells in the human endometrium and decidua have been reported by a number of research groups. These cells reside in both basal and functional layers of the endometrium and can be identified and isolated even from menstrual blood.It was recently demonstrated that endometrial stem/progenitor cells can induce proliferation and this report substantiates the hypothesis on the role of the stem cells in endometrial regeneration[4].The indisputable biological purpose of the endometrium is to secure the successful development of pregnancy. Embryo implantation is only possible for a short period of time when the hostile uterine lining transforms to a hospitable surface to accept the embryo.The newly acquired capacity of the endometrium to welcome the embryo is termed "endometrial receptivity" and it is viewed as a dynamic process of genotypic and phenotypic changes of the endometrial cells. The result is that they are capable of participating in twoway cross-talk with the embryo which may or may not lead to successful opposition, attachment, penetration and implantation and possibly development and growth of a viable conceptus[5,3]. The molecular mechanisms behind this complex and sophisticated process have been studied using animal models and knock-out (KO) mouse studies have positively identified genes for receptivity (leukemia inhibitory factor-LIF,Homeobox protein X3), responses to the embryo (Cyclooxygenase 2-COX 2) and decidualization (Interleukin 11 Receptor-IL-11R).Additional information has derived from in vitro studies with human endometrial cells and explants cultures, human trophoblasts, and placental explant cultures.Members of the cell adhesion molecules family (integrins, etc.) are expressed on the surface of the epithelial cells during the WOI in humans. Extensive studies are being currently carried out on the timed restricted expression of a number of molecules such as mucin (MUC-1), trophinin, Lselectin, Wingless (Wnt) family members, etc. in reference to the possibility of using them as biomarkers for endometrial receptivity[2].In addition to exploring the value of endometrial secretion analysis, N. Macklon has employed a human co-culture model, consisting of decidualized endometrial stromal cells and single hatched blastocysts to identify the soluble factors involved in implantation and to correlate these to embryo development.The cytokines and chemokines produced and secreted by the endometrial cells have been discussed in an extensive review.It is pointed out that numerous cytokines such as IL-11, LIF, IL-15, IL-1 and members of the superfamily of the transforming growth factor (TGF) are important factors in establishing the optimal interactions between the embryo and the endometrium[6]. COX-2 (cyclooxygenase-2), EGF (epidermal growth factor) and LIF (leukemia inhibiting factor) Schematic diagram of the implantation process. Exposure to seminal plasma (SP), the fluid component of ejaculate produced by seminal vesicles, may play a beneficial role in implantation[2].

SP, which contains paternal alloantigens and high concentrations of cytokines, growth factors and prostaglandins, induce a state of active immune tolerance, essential for the embryo to be implanted.Animals that become pregnant through artificial insemination or embryo transfer without being exposed to SP have substantially lower rates of implantation than those exposed to SP[7].Excision of the seminal vesicle glands from males diminishes the tolerance-inducing effect of mating, while the vasectomy to remove sperms from the seminal fluid does not substantially impact the response[8]. These findings have led to the conclusion that the use of SP or its ingredients may stimulate endometrium in IVF cycles in order to improve implantation rates.Several studies have investigated the role of SP in implantation with controversial results, some reporting benefits, whereas others showing no effect $[\mathbf{5 , 3 , 8 ]}$. The purpose of this study is to investigate whether intrauterine application of SP at the time of the ovum pick-up has any influence on the pregnancy rates in IVF or not.

\section{Subjects and Methods:-}


This a randomized,-controlled, a double-blind clinical study investigating superiority of Seminal Plasma administration at the time of oocyte pick-up.

\section{Setting;}

MIRU,miniauniversity,MIVF.

All couples undergoing IVF or ICSI treatment between January 2010 and December 2013 were considered for enrolment in the trial.Of these, 400 women were eligible for enrolment and consented to participate in the study. Participation was limited to one treatment cycle per couple.Patients were included and randomized at the first consultation in our center, before the start of the treatment. Concealed allocation by computerized randomization. Eligible patients were categorized into two groups. Group A who inseminated by seminal plasma and Group B (placebo group).

The exclusion criteria included the presence of hepatitis B, C, leukocytopenia or other signs of infection and men with $<300 \mu \mathrm{l}$ of SP.

The study was approved by the local ethical committee and each patient's approval was given by written consent. Stimulation was performed using the long agonist protocol, pituitary down-regulation was done using GnRH agonist 0.1 IU Leuprolide acetate S.C. (Leucine, Abbviepharmaceutical) starting in the luteal phase of the previous cycle. After down-regulation of the pituitary gland, patients received in addition either 150-300 IU of recombinant FSH (GonalFw, Merck Serono) or highly purified HMG (Fostimon, IBSA pharmaceutical, Switzerland) per day.Once an adequate ovarian response had been confirmed, 10000 IU of urinary hCG (Choriomon, IBA pharmaceutical, Switzerland) was used to trigger ovulation. Ovum pick up was done 34-36 h after hCGadministration under sedation. Fertilization was achieved by ICSI. Day 5 grade 1 embryos were transferred back into the uterus. Luteal support was done using vaginal progesterone (Prontogest 400 bid, IBSA pharmaceutical, Switzerland).

\section{Preparation and application of SP:-}

Semen samples were obtained before follicle aspiration by masturbation from the patient's partner and were collected in sterile flasks. SP was extracted by centrifugation of ejaculates at $600 \mathrm{~g}$ for $15 \mathrm{~min}$. The second round of centrifugation was performed at $10000 \mathrm{~g}$ for $15 \mathrm{~min}$ to extract all spermatozoa.

SP was analyzed several times to exclude remaining contamination with spermatozoa. None of the samples contained any spermatozoa after centrifugation.

Then $400 \mu \mathrm{l}$ of supernatant were diluted with $1600 \mu \mathrm{l}$ of sterile sodium chloride and mixed. From this solution, 1500 $\mu \mathrm{l}$ was stored in $2 \mathrm{ml}$ syringes at $-20^{\circ} \mathrm{C}$ (serum). For the placebo, $1500 \mu \mathrm{l}$ of sterile sodium chloride was also stored in $2 \mathrm{ml}$ syringes at $-20^{\circ} \mathrm{C}$.

Blinding of the samples was achieved by using a placebo (sodium chloride), which could not be differentiated from SP due to the same optical appearance.

An insemination catheter was introduced just at the end of the cervix and $1500 \mu \mathrm{l}$ SP or placebo was injected.

\section{Statistical Analysis:-}

Data were analyzed using Statistical Package for the Social Sciences (SPSS), Version 19. Quantitative data were presented as a range, mean and standard deviation, qualitative data presented as a frequency distribution. Chi-square test, independent sample t-test, ANOVA, and correlation test were used. The probability of less than 0.05 was used as a cut-off point for all significant tests.

\section{Results:-}

Table I shows the demographic criteria of the two groups.400 participants were similar in their characteristics so there is no statistical difference between the study and the placebo groups. 


\begin{tabular}{|c|c|c|}
\hline & Group (A) & Group (B) \\
\hline Mean Age (Years) & $31(23.2: 40.1)$ & $32.1(21.2: 40.9)$ \\
\hline \multicolumn{3}{|l|}{ Cause of infertility } \\
\hline \multirow{5}{*}{$\begin{array}{ll}\text { - } & \text { Male Factor } \\
\text { - Tubal Factor } \\
\text { - Endometriosis } \\
\text { - PCO } \\
\text { - Unexplained }\end{array}$} & 52 & 63 \\
\hline & 30 & 17 \\
\hline & 67 & 43 \\
\hline & 18 & 57 \\
\hline & 33 & 20 \\
\hline \multicolumn{3}{|l|}{ Type of infertility } \\
\hline \multirow{2}{*}{$\begin{array}{l}\text { - Primary } \\
\text { - Secondary }\end{array}$} & 113 & 141 \\
\hline & 87 & 69 \\
\hline No. of ICSI failure & $2.3(1-3)$ & $1.7(0-2)$ \\
\hline
\end{tabular}

Table 2 shows outcomes and embryological data of both groups.There is no statistical difference in the pregnancy rate in the two groups. Also, the number of embryos transferred were similar in both groups so there is no statistical difference between the study and the placebo groups.

\begin{tabular}{|c|c|c|c|}
\hline & Group (A) & Group (B) & P-Value \\
\hline $\begin{array}{c}\text { Median No. of } \\
\text { Transferred embryos }\end{array}$ & $2.71(1: 3)$ & $2.89(1: 4)$ & - \\
\hline $\begin{array}{c}\text { Clinical Pregnancy } \\
\text { Rate }\end{array}$ & $\mathbf{3 9 . 2 \%}$ & $\mathbf{3 1 . 9 \%}$ & $0.69 \mathrm{NS}$ \\
\hline Implantation rate & $\mathbf{5 7 . 4 \%}$ & $49.7 \%$ & $0.39 \mathrm{NS}$ \\
\hline
\end{tabular}

\section{Discussion:-}

This study disputes the hypothesis that intrauterine instillation of SP at the time of oocyte retrieval increases pregnancy rate. We found that there is no difference in the pregnancy rate between the two groups (39.2\% in group A versus $31.9 \%$ in group B p=value 0.69 ) which is statistically insignificant. Also, we found that there is no difference in the implantation rate between the two groups (57.4\% In group A versus $49.7 \%$ in group B p=value 0.39) which is statistically insignificant. In agreement with us, Qasim who found that application of SP in the vagina during intrauterine insemination does not improve the pregnancy rate [9].Also, the same results found by Fishel who declare that there are no differences in the pregnancy rates between the two groups(32\% versus $33 \%$ and $21 \%$ versus $17 \%$ ) [10].Similarly, VonWolff applied thawed SP at the time of OPU during IVF-ICSI cycles and found a non-significant increase in the pregnancy rate $(37.3 \%$ versus $25.7 \%)$ [11].In contrast, Bellinge who deposited semen in the vagina of patients undergoing IVF at the time of the oocyte fertilization and found an implantation rate of $53 \%$, compared with $23 \%$ in the control group [12].Also, Coulam and Stern performed a placebo-controlled clinical trial, depositing into the vagina capsules containing SP or placebo. They described an implantation rate of 80 $\%$ in the SP group, compared with $67 \%$ in the placebo group [13].Therefore, the previous studies are diverse and difficult to compare.

These different results of the previous studies could be explained by the followings

Firstly, different sites of insemination whether cervical, vaginal or intrauterine. In the study published in 2009, pure SP was installed into the cervix, which is very close to the physiological situation during sexual intercourse. In this study, SP was injected into the uterine cavity, which is nonphysiological. Gutsche et al. found that seminal plasma induces endometrial inflammatory responses only vitro in human [3], whereas cervical inflammatory responses 
following exposure to ejaculate have been demonstrated in vivo [8]. Also, in vitro experiments demonstrated that this effect is due to SP and not due to semen [14].Second, trauma induced by the catheter while introduced through the cervix into the uterus may increase the pregnancy rate without any pathophysiological effect.This matches several studies that have demonstrated an increased pregnancy rate after the endometrial injury before IVF treatment (Nastri et al., 2012). This effect was not seen after endometrial injury at the time of follicle puncture.

However, as the introduction of a transfer catheter possibly also causes some cervical trauma, it might be speculated that this would have a positive effect in our new study, although in reality such an effect was not observed. Finally, there is the possibility that intracavitary treatment with SP does not have any clinically relevant immunomodulatory effects on the endometrium at all or that the dilution of SP reduced the effect to such an extent that it was not pronounced enough to have a substantial effect on endometrial function. Indeed, the present study suggests that SP installation into the uterine cavity is unlikely to increase the chance of clinical pregnancy, at least not by a rate $\geq 14 \%$ as initially hypothesized.

In conclusion besides the quality of the embryo, endometrial receptivity plays an important role in the establishment of pregnancy. Endometrial functional should be considered of great relevance in a physiological context, e.g. spontaneous conception in a natural cycle and implantation in assisted conception.Finally, there is the possibility that insemination of SP does not have any clinically relevant immunomodulatory effects on the endometrium at all, or have a substantial effect on endometrial function.Intrauterine instillation of diluted SP at the time of ovum pickup is unlikely to increase the pregnancy rate.

In conclusion, the results of this study cast doubt on the validity of the concept that SP increases endometrial receptivity and thus implantation in humans.

Clinically relevant differences cannot reliably be excluded based on this single study,

Finally, the answer to the question about the role of seminal plasma insemination at the time of OPU is still unclear, therefore further studies are still needed, with a larger number of patients; this will further justify the exact place of IU insemination of SP in modifying the endometrial function.

\section{Conflict of interest:-}

We declare no conflict of interest.

\section{Acknowledgement:-}

We have not received any funding from any corporate body or pharmaceutical company.

\section{References:-}

1. von Wolff M, Ro"sner S, Tho"ne C, Pinheiro RM, Jauckus J, Bruckner T, BiolchiAlia A, Strowitzki T. Intravaginal and intracervical application of seminal plasma in vitro fertilization or intracytoplasmic sperm injection treatment cycles-a double-blind, placebo-controlled, randomized pilot study. FertilSteril 2009;91:167172.

2. Nastri CO, Gibreel A, Raine-Fenning N, Maheshwari A, Ferriani RA, Bhattacharya S, Martins WP. Endometrial injury in women undergoing assisted reproductive techniques. Cochrane Database Syst Rev.2012; 11:CD009517.

3. Gutsche S, von Wolff M, Strowitzki T, Thaler CJ. Seminal plasma induces mRNA expression of IL-1b, IL-6 and LIF in endometrial epithelial cells vitro. Mol Hum Reprod 2003;9:785-791.

4. Robertson SA, Prins JR, Sharkey DJ, Moldenhauer LM. Seminal fluid and the generation of regulatory T cells for embryo implantation. Am J ReprodImmunol 2013;69:315-330.

5. Chicea R, Ispasoiu F, Focsa M. Seminal plasma insemination during ovum pickup- a method to increase pregnancy rate in IVF/ICSI procedure. A pilot randomized trial. J Assist Reprod Genet 2013;30:569-574.

6. Doyle U, Sampson N, Zenzmaier C, Schwa"rzler P, Berger P. Seminal plasma enhances and accelerates progesterone-induced decidualisation of human endometrial stromal cells. ReprodFertil Dev 2012;24:517-522.

7. El-Toukhy T, Sunkara SK, Coomarasamy A, Grace J, Khalaf Y. Outpatient hysteroscopy and subsequent IVF cycle outcome: a systematic review and meta-analysis. Reprod Biomed Online 2008;16:712-719. 
8. Sharkey DJ, Tremellen KP, Jasper MJ, Gemzell-Danielsson K, Robertson SA. Seminal fluid induces leukocyte recruitment and cytokine and chemokine mRNA expression in the human cervix after coitus. J Immunol 2012; 188:2445-2454.

9. Qasim SM, Trias A, Karacan M, Shelden R, Kemmann E. Does the absence or presence of seminal fluid matter in patients undergoing ovulation induction with intrauterine insemination? Hum Reprod. 1996;11(5):1008-10.

10. Fishel S, Webster J, Jackson P, Faratian B. Evaluation of high vaginal insemination at oocyte recovery in patients undergoing in vitro fertilization. FertilSteril. 1989;51:135-8.

11. Von Wolff M, Rosner S, Thone C, Pinheiro RM, Jauckus J, Bruckner T, et al. Intravaginal and intracervical application of seminal plasma in in vitro fertilization or intracytoplasmic sperm injection treatment cycles - a double-blind, placebo-controlled, randomized pilot study. FertilSteril. 2009;91(1):167-72.

12. Bellinge BS, Copeland CM, Thomas TD, Mazzucchelli RE, O'Neil G, Cohen MJ. The influence of patient insemination on the implantation rate in an in vitro fertilization and embryo transfer program. FertilSteril. 1986;46:25-6.

13. Coulam CB, Stern JJ. Effect of seminal plasma on implantation rates. Early Pregnancy. 1995;1:33-6.

14. Sharkey DJ, Macpherson AM, Tremellen KP, Mottershead DG, Gilchrist RB, Robertson SA. TGF-b mediates proinflammatory seminal fluid signaling in human cervical epithelial cells. J Immunol 2012b;189: 1024-1035.

15. Nastri CO, Gibreel A, Raine-Fenning N, Maheshwari A, Ferriani RA, Bhattacharya S, Martins WP. Endometrial injury in women undergoing assisted reproductive techniques. Cochrane Database Syst Rev 2012; 11:CD009517. 